\title{
3D Autonomous Navigation of Quadrotor using Redundant Flight Controllers: Comparison study
}

\author{
Y. BOUZID, Y. Bestaoui /IBISC, Université d'Evry- \\ Val-d'Essonne, Université Paris-Saclay \\ Evry, France \\ yasseremp@gmail.com \\ Yasmina.Bestaoui@ufrst.univ-evry.fr
}

\begin{abstract}
In this paper, two redundant controllers are proposed in order to boost the capabilities of the popular feedback linearization flight controller scheme. The first one is based on the sliding mode framework whilst the second one is built upon the Model Free Control (MFC) theory. An in-depth discussion is highlighted with detailed evaluation in terms of performance, consumed energy, and robustness by considering several scenarios and using several metrics. The numerical simulations have shown satisfactory results using nominal system model or disturbed model through an application to a small Vertical Take-Off and Landing (VTOL) quadrotor.
\end{abstract}

Keywords-Trajectory tracking, Autonomous vehicle, Control performance, Robustness.

\section{INTRODUCTION}

The popularity of quadrotor Unmanned Aerial Vehicle (UAV) has grown tremendously in academic, governmental, industrial missions as for instance: monitoring, mapping, detection, as well as search and rescue missions [1]. Duo to its noteworthy properties, particularly the structural simplicity, rapid maneuverability and stable hovering capability, the quadrotor is considered as a good case study to design and analyze flight control strategies.

The linearization of a highly coupled nonlinear model notably degrades the performance of control, and in such situation, the linear control algorithms fail to control the system at points rather than the operating point [2-3]. This pushes the researchers toward alternative strategies to bring more improvements. In the two last decades, a broad range of nonlinear flight control techniques have been studied where the performances of control are clearly improved (see [4-6]). However, implementing a nonlinear control technique requires a deep study of the system nonlinearities, where extracting the complete model is almost an impossible task to be achieved. Besides, disturbances could lead to partial or complete changes in the system dynamics.

Going beyond these motivations, the present paper is interested by proposing redundant controllers. Thus, two alternatives are considered that are used for the stabilization as well as for the 3D trajectory tracking. They boost the feedback linearization controller performance especially the robustness level with moderate computational complexity. The first approach employs an input dependent sliding mode surface. This technique is called Dynamic Sliding Mode Control

\author{
H. Siguerdidjane / L2S, CentraleSupélec \\ Université Paris-Saclay \\ Gif-sur-yvette, France \\ Houria.Siguerdidjane@centralesupelec.fr
}

(DSMC) whilst the second technique is based on the se-called Model Free Control (MFC). Throughout the paper, a performance assessment is presented via results of several illustrations, scenarios and numerical simulations, with complementary comments on the drawbacks and advantages of each strategy. Particular attention is paid to the tracking accuracy and the energy consumption of each control strategy considering some performance criteria, such as: Integral Squared Error (ISE) and Integral Squared Control Input (ISCI).

This paper is structured as follows. The second section overviews the dynamics and the control architecture of the VTOL quadrotor. Section 3 introduces the design of the nonlinear control approaches. In Section 4, the results from numerical simulations test the effectiveness of the proposed control strategies under different operating conditions. The final section gives some conclusions.

\section{VEHICLE DYNAMICS \& CONTROL ARCHITECTURE}

The general dynamic model of a quadrotor has been presented in a number of papers and will not be discussed here again (see [5], [7] or [8]). The system operates in two coordinate frames: the earth fixed frame $R_{0}(O, X, Y, Z)$ and the body fixed frame $R_{1}\left(O_{1}, X_{1}, Y_{1}, Z_{1}\right)$ (see Figure 1$)$. The simplified dynamic model, in terms of position $\chi=(x, y, z)^{T} \in \mathbb{R}^{3}$ and rotation $\left.\eta=(\varphi, \theta, \Psi)^{T} \in\right]-\frac{\pi}{2}, \frac{\pi}{2}[\times]-\frac{\pi}{2}, \frac{\pi}{2}[\times]-\pi, \pi[$, is written as:

$$
\ddot{\xi}=\left[\begin{array}{c}
\frac{u_{x}}{m} \\
\frac{u_{y}}{m} \\
-g+\frac{u_{z}}{m} \\
\dot{\theta} \dot{\Psi}\left(\frac{I_{y}-I_{z}}{I_{x}}\right)+\frac{u_{\varphi}}{I_{x}} \\
\dot{\varphi} \dot{\Psi}\left(\frac{I_{z}-I_{x}}{I_{y}}\right)+\frac{u_{\theta}}{I_{y}} \\
\dot{\varphi} \dot{\theta}\left(\frac{I_{x}-I_{y}}{I_{z}}\right)+\frac{u_{\Psi}}{I_{z}}
\end{array}\right]
$$

where $s_{(.)}$and $c_{(.)}$are abbreviations for $\sin ($.$) and \cos ($. respectively, $\mathrm{m}$ is the mass, $\mathrm{g}$ is the gravity acceleration, $I=$ $\operatorname{diag}\left(I_{x}, I_{y}, I_{z}\right)$ is the diagonal inertia matrix and $\tau=$ $\left(u_{\varphi}, u_{\theta}, u_{\Psi}\right)^{T}$ is the control torque vector. $\xi=(\chi, \eta)^{T}$ denotes the output vector. $u_{x}, u_{y}$ and $u_{z}$ are virtual inputs given by

$$
\left\{\begin{array}{l}
u_{x}=u_{1}\left(c_{\Psi} s_{\theta} c_{\varphi}+s_{\Psi} s_{\varphi}\right) \\
u_{y}=u_{1}\left(s_{\Psi} s_{\theta} c_{\varphi}-c_{\Psi} s_{\varphi}\right) \\
u_{z}=c_{\theta} c_{\varphi} u_{1}
\end{array}\right.
$$

where the following equality must be verified 
$u_{x}^{2}+u_{y}^{2}+c_{\theta}^{2} c_{\varphi}^{2}=1$

with $u_{1}$ denotes the total thrust. From system (2), we get

$\left\{\begin{array}{l}\varphi_{r}=\arcsin \left(\frac{u_{x} \sin \Psi_{r}-u_{y} \cos \Psi_{r}}{u_{1}}\right) \\ \theta_{r}=\arcsin \left(\frac{u_{x} \cos \Psi_{r}+u_{y} \sin \psi_{r}}{u_{1} \cos \varphi_{r}}\right)\end{array}\right.$

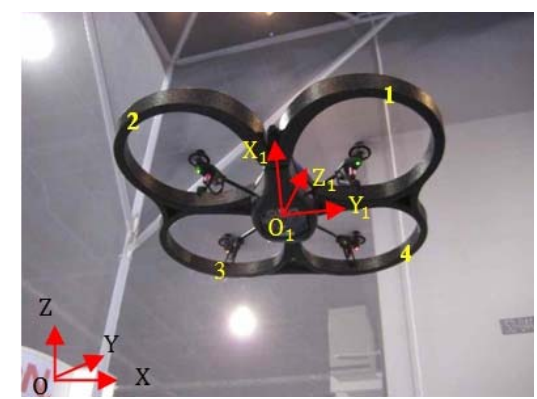

Figure 1. Quadrotor in experimentations.

In the position control, $\mathrm{x}$ and $\mathrm{y}$ are controlled through the two virtual inputs $\left(u_{x}, u_{y}\right)$ that push the system to reach the prescribed references $x_{r}$ and $y_{r}$ respectively and allow to generate the reference angles $\left(\varphi_{r}, \theta_{r}\right)$ via equation (4). The Euler angles $\eta$ are controlled by the torque vector $\tau$, whereas the altitude is controlled by $u_{1}$. This control architecture is described by Figure 2 .

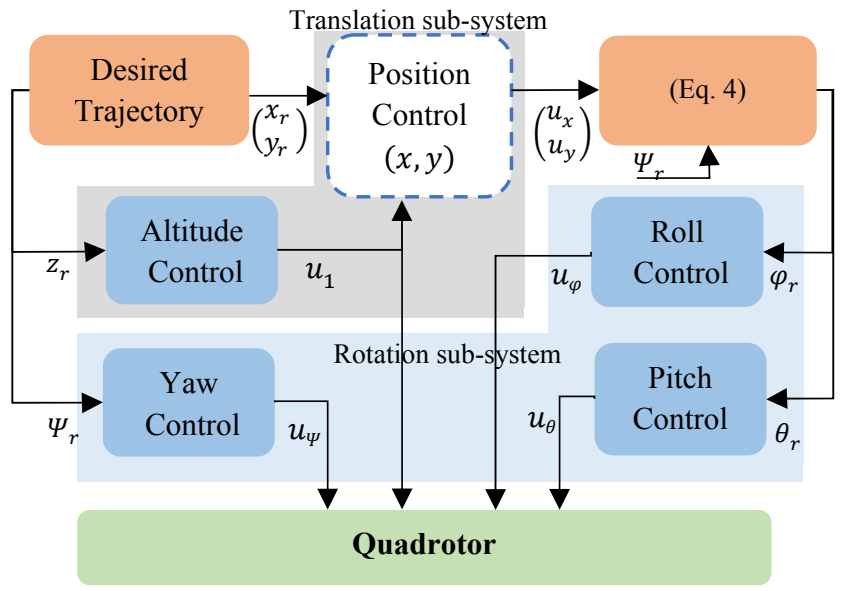

Figure 2. Quadrotor control architecture.

The Multi-Input Multi-Output (MIMO) system (1) may be written under the form

$M \ddot{X}=\Phi(X)+u_{f}$

where $X \in D_{X} \subset \mathbb{R}^{n}$ is an $n$-dimentional state vector, $u_{f} \in$ $\mathcal{U} \subset \mathbb{R}^{n}$ is the input vector, $\Phi: D_{X} \rightarrow \mathbb{R}^{n}$ is $n$-dimentional vector function sufficiently smooth on a domain $D_{X} \subset \mathbb{R}^{n}$ and $M$ is the diagonal matrix that contains the inertia and mass elements. The same MIMO system (1) also can be divided into a set of Single-Input Single-Output (SISO) systems affine in the control written as

$\ddot{\xi}=f_{\xi}\left(\xi, w_{f}\right)+\left.\kappa_{\xi} u_{\xi}\right|_{\xi=\{x, y, z, \varphi, \theta, \Psi\}}$

where $M, \Phi(),. f_{\xi}($.$) and \kappa_{\xi}$ can be identified from the overall dynamic model (1). $w_{f}$ may be considered as time varying term. The parameters of the system UAV used in this study are displayed in Table 1.

Table 1. Quadrotor parameters.

\begin{tabular}{|l|c|c|c|}
\hline$m(\mathrm{~kg})$ & 0.429 & $I_{y}\left(\mathrm{~kg} \cdot \mathrm{m}^{2}\right)$ & 0.0029 \\
\hline$I_{x}\left(\mathrm{~kg} \cdot \mathrm{m}^{2}\right)$ & 0.0022 & $I_{z}\left(\mathrm{~kg} \cdot \mathrm{m}^{2}\right)$ & 0.0048 \\
\hline
\end{tabular}

\section{FLIGHT CONTROLLERS DESIGN}

Feedback linearization is a popular control technique. However, it requires an accurate estimation of the current state, which is not obvious in practice. In addition, external disturbances may lead to the instability of the system. Therefore, the capabilities of this controller are boosted by employing additional control loops. In this section, we present two nonlinear strategies.

\section{A. Dynamic sliding mode}

In this sub-section, we propose to design an input dependent DSMC for 3D trajectory tracking of quadrotor that boosts the feedback linearization control law capabilities. Unlike the classical Sliding Mode Control (SMC), the DSMC employs, as sliding surface, the system input. In reference [9], a redundant control scheme was proposed for asymptotic space vehicle stabilization and has shown very promising results with respect to sudden failures in the designed feedback loop. Herein, we try to supplement this study where the inputdependent sliding surface is employed to improve the robustness level with respect to external disturbances. In function of the tracking errors, model (5) is written as

$M \dot{E}=-\Phi(X)-u_{f}+M \dot{X}_{r}$

where $E=X_{r}-X$. to

Appling the classic feedback linearization controller leads

$u_{f}=-\Phi(X)+M\left(\dot{X}_{r}+K_{1} E+K_{2} \dot{E}\right)$

$u_{f}$ is considered as smooth control input that insures the asymptotic convergence of the tracking error toward the origin with $K_{1}>0$ and $K_{2}>0$. Now we consider a disturbed model defined as

$(M+\delta M) \dot{E}=-(\Phi(X)+\delta \Phi(X))+M \dot{X}_{r}-u$

where $\delta \mathrm{M}$ and $\delta \Phi($.$) denote the mismatch between nominal$ model (7) and disturbed one (9). Herein, $u$ is the novel control input needed to ensure the asymptotic stability of the disturbed model. Therefore, we consider a supplementary effort with respect to the nominal system, $u=u_{f}+\delta u$ to deal with the disturbances. Thus, system (9) becomes

$M \dot{E}=-\Phi(X)+M \dot{X}_{r}-u_{f}+\delta u+\Delta(X)$

with $\Delta(X)=-\delta \Phi(X)-\delta M \dot{E}$. So the idea is to compensate, or at least overtaken, the unknown mismatch term $\Delta(X)$ using the supplementary effort $\delta \mathrm{u}$. In general, the disturbance term $\Delta(X)$ may represent a neglected dynamic, perturbation, uncertainties, etc. It is unknown but bounded, $\|\Delta(X)\| \leq d$ where $d$ is a positive constant.

Usually, the basis in the sliding mode scheme is the definition of a sliding surface. Contrary to the common case, a surface vector is defined as

$s=u-u_{f}=\delta u$

Such sliding surface vector $s \in \mathbb{R}^{\mathrm{n}}$ has the interpretation that any mismatch between the nominal model and the 
disturbed model yields a deviation in the input signal. If a control law drives the trajectories such that $s=0$ holds true, then the perturbation term is totally rejected and the tracking errors converge toward the origin. Considering a positive definite Lyapunov candidate function

$V=\frac{1}{2} s^{T} S$

The first time derivative is

$\dot{V}=s^{T} \dot{S}$

Note that the reachability condition of sliding mode control is ensured if $\dot{V}$ is negative definite $(\dot{V}<0)$. In the following, a discontinuous dynamics are introduced by

$\dot{s}=-W \gamma(s)=-W \gamma(\delta u)$

where $W$ is an arbitrary strictly positive diagonal matrix and $\gamma($.$) is an operator acts on the surfaces. The ideal sliding mode$ holds for an operator defined by a Signum function $(\gamma(a)=$ $\operatorname{sign}(a))$. In the practice, it is recommended to use smother functions in order to reduce the chattering problem.

Substituting equation (11) into (14) and by straightforward integration, it comes that

$$
u=-\int_{0}^{t}\left(W \gamma(s)-\dot{u}_{f}\right) d \tau+u(0)
$$

So

$$
u=u_{f}-\int_{0}^{t} W \gamma(s) d \tau+u(0)
$$

This new controller form, as the classic sliding mode controller, is highly insensitive to external perturbation and to modeling errors. In view in this new formulation, the scheme thus operates as a parallel feedback with high gain loop that enforces the basic smooth feedback control. If the smooth portion has robustness capabilities with respect to certain class of perturbations, the proposed controller that involves, in addition, a discontinuous integral portion has very high robustness features and performances. The integral term plays a compensator role. For this raison, the matrix $W$ need to be tuned carefully according to the boundaries of the disturbance. This controller is depicted in the block diagram of Figure 3.

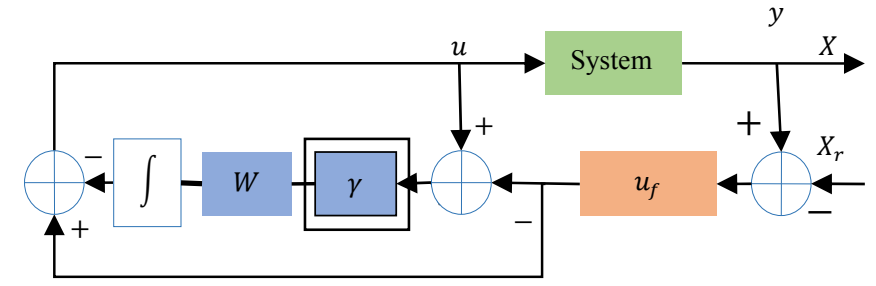

Figure 3: DSMC diagram.

\section{B. Model free based control}

In fact, the control of a system with a model free technique has been used, since many decades, on the basis of fuzzy logic control or the more popular one for linear systems through Ziegler Nichols method [10]. Its use as basis of control will allow compensating the uncertainties as well as other disturbances. This is due to the anticipation nature of the unmodeled dynamics and system uncertainties, which makes the control possible even with the presence of disturbances. It is employed in many applications such as mobile robot [11], DC/DC converters [12] and active magnetic bearing [13].

The fact that this strategy requires some inversion (see Eq (22)), which need a deep analysis and for the sake of simplicity, we consider a class of nonlinear SISO systems for $t \in[0, \infty)$ given by

$y^{(n)}(t)=f\left(\zeta, w_{f}\right)+b u(t)$

where $\zeta=\left(y(t), \dot{y}(t) \ldots, y^{(n-1)}(t)\right)^{\mathrm{T}} \in D_{\zeta} \subset \mathbb{R}^{n}$ is an $n$ dimentional state vector, $u \in \mathcal{U} \subset \mathbb{R}$ is a scalar input, $y \in$ $D_{y} \subset \mathbb{R}$ is a scalar output, $f():. D_{\zeta} \rightarrow \mathbb{R}$ is multi-variable nonlinear function. $b$ is the input parameter and $w_{f}$ is time varying known terms.

In the literature, the use of MFC is linked to the linear PID or PD controller under the name intelligent-PID ( $i$ PID) and $i$ PD for $n=2$ or $n=1$ respectively. Moreover, the existing MFC techniques consider only the input-output signals in order to design the controller. However, we have, currently, a nominal model for the controlled system even if it is an approximated version that can be exploited to synthesize the controller. Therefore, for a significant improvement, in this paper, we propose to use the MFC with a nonlinear feedback linearization technique rather than using it with the classical linear controllers (PI or PID) by considering the available nominal model where the MFC principle is investigated for the unknown dynamics of the system.

The input-output relationship of the anticipated disturbed model may be represented by the form

$y^{(v)}=f\left(\zeta, w_{f}\right)+b u+\Delta(\zeta, u)$

Motivated by system model (17), the mismatch term could be written as

$\Delta(\zeta, u)=\tilde{f}(\zeta)+\kappa u$

where $v$ is the order of the anticipated model, $\kappa$ is an input positive scaling factor and $\tilde{f}(\zeta)$ is the disturbance dynamics.

From equations (18) and (19), it comes that

$y^{(v)}=f\left(\zeta, w_{f}\right)+(b+\kappa) u+\tilde{f}(\zeta)$

According to the MFC strategy, the estimated term $\tilde{f}(\zeta)$ is continuously updated. From (20), we define

$\tilde{f}(\zeta)=\hat{y}^{(v)}-(b+\kappa) \hat{u}-f\left(\zeta, w_{f}\right)$

Thanks to knowledge of the past input $\hat{u}$ and $\kappa$ where $\hat{y}^{(v)}$ denotes the $v^{\text {th }}$ derivative of the measure $y$ in the previous time interval, the value of $\tilde{f}(\zeta)$ is estimated. This estimation is of course valid for a short period of time and it should be continuously updated on every iteration of the closed loop controller. This updated value injects the required change in the control input where the control input for MFC strategy is written as follows

$u=\frac{-\tilde{f}(\zeta)+u_{f}}{b+\kappa}$

where $u_{f}$ is an auxiliary input. Substituting (22) into (20) yields

$y^{(v)}=f\left(\zeta, w_{f}\right)+u_{f}$

$u_{f}$ should be selected in order to push the tracking error of system (23) toward the origin. The feedback linearization technique leads to

$u_{f}=y_{r}^{(v)}-f\left(\zeta, w_{f}\right)+k_{1} e+k_{2} \dot{e}$

where $e=y-y_{r}$ denotes the tracking error, $y_{r}$ denotes the reference trajectory and $u_{f}$ is the nominal feedback that should 
be selected in order to push the tracking error of system (23) toward the origin. $k_{1}$ and $k_{2}$ are two positive constants. This control structure is depicted in Figure 4.

The proposed approach presents several benefits such as the simplicity of its structure. Moreover, it exhibits a good level of robustness with self adaption in case of uncertainties and boosts the robustness capabilities level of the system.

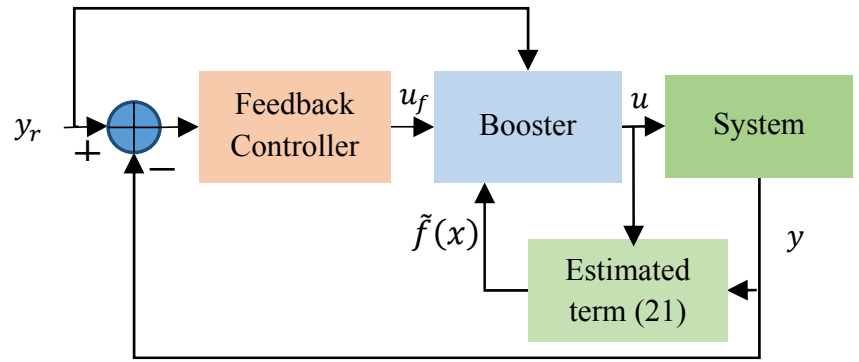

Figure 4. MFC principle scheme.

\section{RESULTS AND DISCUSSION}

In this section, we test the effectiveness of the proposed controllers not only in the ideal case but also in the presence of different disturbances. For the sake of further comparison, we follow the same protocol and we respect the same conditions. The control parameters are tuned, using Genetic Algorithms (GA), in the ideal case then kept for the entire proposed scenarios where the objective is to reduce the steady state errors. Thus, the fitness function is given by

$I S E=\frac{1}{t_{f}-t_{i}} \int_{t_{i}}^{t_{f}}\left(\begin{array}{c}\chi_{r}-\chi \\ \eta_{r}-\eta\end{array}\right)^{T}\left(\begin{array}{c}\chi_{r}-\chi \\ \eta_{r}-\eta\end{array}\right) d t$

where $t_{i}$ and $t_{f}$ denote the initial and the final instants of optimization respectively.

The overall system dynamics and control laws are implemented on Matlab program. The total simulation time is 40 seconds and the sampling time is 0.01 seconds. We simulate the response of the quadrotor using the system available parameters (see Table 1). We consider many scenarios in order to be closer to the realistic situation:

- Basic scenario: In this scenario, after the take-off, the quadrotor tracks a square reference trajectory $(2 \mathrm{~m} \times 2 \mathrm{~m})$ that is described as

$\sigma(t)= \begin{cases}0 & \text { when } 0 \leq t \leq t_{1} \\ L_{r} \frac{\left(t-t_{1}\right)^{5}}{\left(t-t_{1}\right)^{5}+\left(T-t+t_{1}\right)} & \text { when } t_{1}<t \leq t_{2} \\ L_{r} & \text { when } t_{2}<t \leq t_{3} \\ L_{r}-L_{r} \frac{\left(t-t_{3}\right)^{5}}{\left(t-t_{3}\right)^{5}+\left(T-t+t_{3}\right)} & \text { when } t_{3}<t \leq t_{4} \\ 0 & \text { when } t_{4}<t \leq t_{f}\end{cases}$

with $T=5$ seconds, $t_{f}=40$ seconds and $L_{r}=2$ meters.

$x_{r}=\sigma(t)$ with $t_{1}=5, t_{2}=t_{1}+T, t_{3}=25, t_{4}=t_{3}+T$

$y_{r}=\sigma(t)$ with $t_{1}=10, t_{2}=t_{1}+T, t_{3}=30, t_{4}=t_{3}+T$

$z_{r}=\sigma(t)$ with $t_{1}=0, t_{2}=t_{1}+T, t_{3}=35, t_{4}=t_{3}+T$

- Parameters uncertainties: The control systems should be capable to tolerate model uncertainties or parameter uncertainties. We suppose that the inertia matrix elements and the aerodynamic coefficients are underrated $50 \%$ of the real values.

- Extra payload: The flight controllers should be able to ensure good performance with good level of robustness even with extra mass. Therefore, in this scenario, the quadrotor is supposed using a heavy camera with additional mass that represents $50 \%$ from the initial mass of quadrotor. The camera is supposed as rigid compact body located at the center of mass of quadrotor.

- Sensor noise: Low cost sensors are usually used in the quadrotors. Thus, the precision is low and the output signals are always noisy. Moreover, the estimation algorithms also cause big errors and drifts. In this scenario, we test the tolerance of controllers to noise that may affect the measured signals. Thus, we add the sensor noise on the states of the system. The expression of the noisy states is

$\tilde{\chi}=\chi+N_{c} \operatorname{rand}($.

$\left\{\tilde{\eta}=\eta+N_{c} \operatorname{rand}()\right.$.

rand(.) is a Matlab function, which generates a random number between 0 and $1 . N_{c}$ is a scale parameter to adjust the level of noise.

- Wind disturbance: Quadrotors are readily affected by external disturbances during the outdoor flight. This scenario is dedicated to test the stability of control systems while encountering environment disturbances, namely gust of wind. Here, we accept that the wind cause the same acceleration intensity on all X,Y,Z-axes. These accelerations are considered as perturbations added to the equations related to the forces in the quadrotor model. Therefore, the disturbed model is expressed as follows

$\ddot{\tilde{x}}=\ddot{x}+a_{x}(t)$

$\ddot{\tilde{y}}=\ddot{y}+a_{y}(t)$

$\ddot{\tilde{z}}=\ddot{z}+a_{z}(t)$

The profile of this acceleration is depicted in Figure 5 and expressed explicitly as

$a_{i}(t)=$

$0 \quad$ when $0 \leq t \leq t_{1}$

$0.8 \sin \left(\frac{\pi(t-30)}{31}\right)+0.4 \sin \left(\frac{\pi(t-30)}{7}\right)+0.08 \sin \left(\frac{\pi(t-30)}{2}\right)+$

$0.056 \sin \left(\frac{\pi(t-30)}{11}\right) \quad$ when $t_{1}<t \leq t_{2}$

0 when $t_{2}<t \leq t_{f}$

with $t_{1}=10$ and $t_{2}=30 i=x, y, z$.

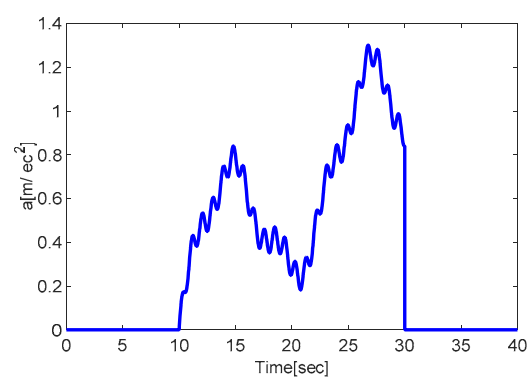

Figure 5. Wind disturbance profile. 
A. Numerical simulations

In this sub-section, we discuss the results in comparison to the feedback linearization controller in order to investigate the improvement and the efficiency of the proposed strategies described in Section III. The control tuning parameters are given in Table 2 . We test the effectiveness of the controller considering all the scenarios explained above. The results for the basic scenario are depicted in Figure 6, Figure 7 and Figure 8. In each figure, we plot separately, the tracking errors of the translations along X,Y,Z-axes, the attitude angles $\varphi, \theta$ and $\Psi$ and the control inputs (global thrust and torques).

Table 2: Feedback linearization control parameters.

\begin{tabular}{|c|c|c|c|c|c|}
\hline$K_{1 x}$ & 23.33 & $K_{1 y}$ & 22.19 & $K_{1 z}$ & 21.77 \\
\hline$K_{2 x}$ & 5.05 & $K_{2 y}$ & 4.86 & $K_{2 z}$ & 4.99 \\
\hline$K_{1 \varphi}$ & 18.77 & $K_{1 \theta}$ & 20.672 & $K_{\Psi}$ & 19.16 \\
\hline$K_{2 \varphi}$ & 4.51 & $K_{2 \theta}$ & 5.00 & $K_{2 \Psi}$ & 4.58 \\
\hline
\end{tabular}

Overall, from the curves obtained in Figure 6, Figure 7 and Figure 8, and through the application of DSMC and MFC respectively, the results are improved in terms of accuracy compared to the classic feedback linearization. The controllers stabilize correctly roll and pitch angles where the quadrotor follows its reference trajectory in good manner. It is seen that the errors are acceptably small with moderate energy consumption.
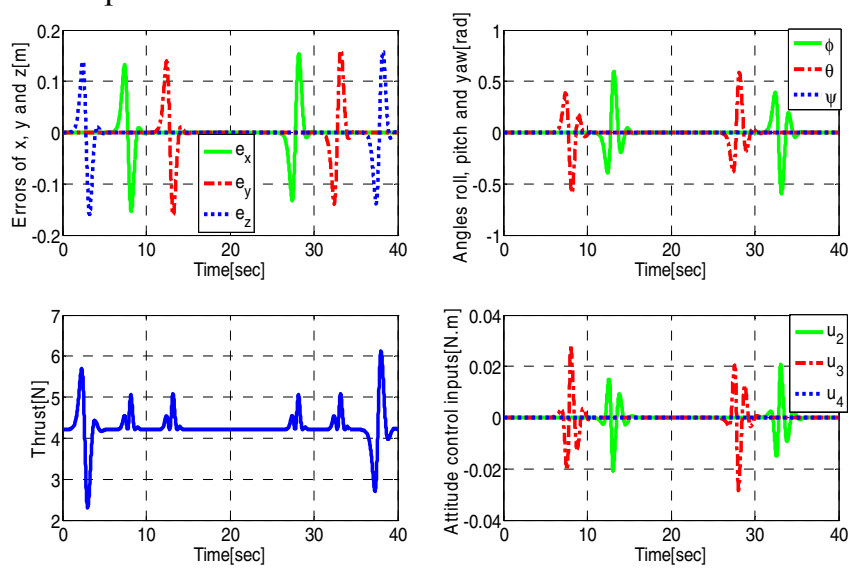

Figure 6. Feedback linearization control in the basic scenario.
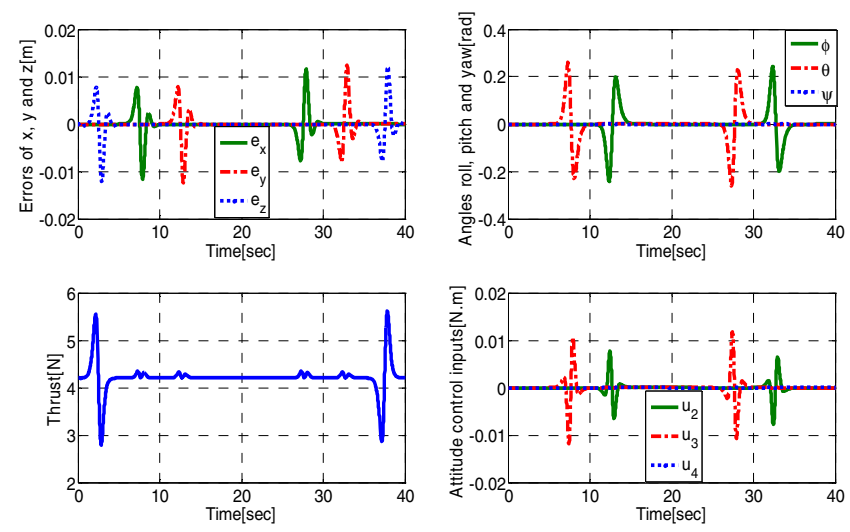

Figure 7. DSMC in the basic scenario.
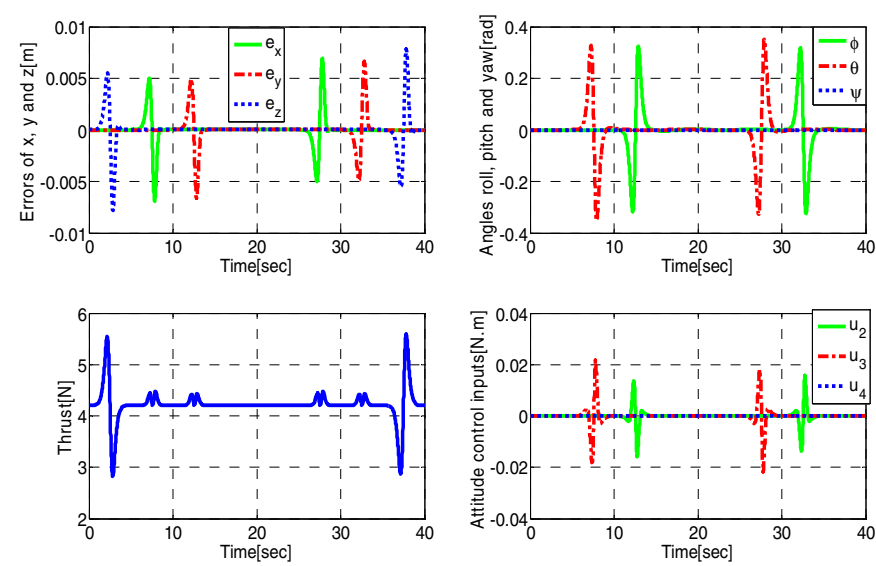

Figure 8. Model free control in the basic scenario.

\section{B. Comparative results}

For the remain of scenarios, we suggest to quantify the obtained results in order to get a close view on the features so deep analysis of the performances. In order to qualify the overall performance of the control strategies, some analysis tools, such as the ISE, the MAE and the ISCI are chosen as metrics for the comparison. They are given by:

- Maximum Absolute Error (MAE): It is measured as: $M A E=\max \left(\left|e_{x}\right|,\left|e_{y}\right|,\left|e_{z}\right|\right)$.

- Integral Square Error (ISE): It is given by

$$
I S E=\int_{t_{0}}^{t_{f}}\left(e_{x}^{2}(t)+e_{y}^{2}(t)+e_{z}^{2}(t)\right) d t
$$

- Integral Squared Control Input (ISCI): It allows to measure the consumed energy using

$$
I S C I=\int_{t_{0}}^{t_{f}} u_{1}(t) d t
$$

The quantified metrics are summarized for each scenario in Table 3.

Table 3: Metrics for each scenario.

\begin{tabular}{|l|c|c|c|}
\hline & FL & MFC & DSMC \\
\hline \multicolumn{4}{|c|}{ Basic Scenario } \\
\hline ISE & 0.11769 & 0.00247 & 0.002312 \\
\hline MAE & 0.161344 & 0.01631 & 0.013604 \\
\hline ISCI & 731.8141 & 717.8735 & 710.57916 \\
\hline \multicolumn{4}{|c|}{ Parameters uncertainties } \\
\hline ISE & 8.347492 & 1.17827 & 8.12000 \\
\hline MAE & 0.79291 & 0.249784 & 0.6240 \\
\hline ISCI & 734.1009 & 719.73 & 2846.59 \\
\hline \multicolumn{5}{|c|}{ Extra payload } \\
\hline ISE & 2.32331 & 0.29793 & 2.05037 \\
\hline MAE & 0.478373 & 0.12467 & 0.31420 \\
\hline ISCI & 1655.32 & 1619.6805 & 1600.25181 \\
\hline \multicolumn{4}{|c|}{ Noise } \\
\hline ISE & 0.13233 & 0.006107 & 0.01724 \\
\hline MAE & 0.171648 & 0.01474 & 0.027246 \\
\hline ISCI & 731.930 & 718.0718 & 710.4363 \\
\hline
\end{tabular}


Wing gust

\begin{tabular}{|l|c|c|c|}
\hline ISE & 0.18039 & 0.00889 & 0.063617 \\
\hline MAE & 0.186976 & 0.023565 & 0.06302 \\
\hline ISCI & 690.6983 & 676.7904 & 665.3723 \\
\hline
\end{tabular}

Obviously, using the DSMC and MFC boosters, the feedback control becomes more accurate (see Table 3). In the basic scenario, the techniques DMSC and MFC exhibit the same performance in terms of accuracy where the MFC consumes more energy. For the remain of scenarios, the DSMC is the less accurate compared to MFC. We also observe that the DSMC consumes less energy while the MFC consumes more energy. The MAE measures the level of overshoot in the system time response, which is not recommended for the quadrotors the fact that physical oscillations may be occurred during the flight. Therefore, Table 3 shows that the best controller is the MFC. In fact, DSMC also allows a damped response compared to the classic feedback linearization controller.

\section{CONCLUSION}

A comparative study between two nonlinear control laws was completed. By introducing an integral action in the DSMC, the steady state errors vanish efficiently. A second proposed novel way to design a MFC is described. This controller differs from the classic one by using a feedback linearization instead of the PID. It raises the performance with respect to structured and unstructured uncertainties.

Numerical simulations have been performed on the nonlinear dynamic model of quadrotor in order to test the effectiveness of the designed control systems. The strong efficiency of the proposed approaches is demonstrated in multiple test scenarios in order to point out the best controller for each case. The settling time is shown to be quite fast and a good level of robustness is ensured with respect to the parameters uncertainties and the external disturbances.

\section{REFERENCES}

[1] T. Tomic et al., "Toward a Fully Autonomous UAV: Research Platform for Indoor and Outdoor Urban Search and Rescue," IEEE Robotics Automation Magazine, vol. 19, no. 3, pp. 46-56, Sep. 2012.

[2] A. L. Salih, M. Moghavvemi, H. A. F. Mohamed, and K. S. Gaeid, "Modelling and PID controller design for a quadrotor unmanned air vehicle," in 2010 IEEE International Conference on Automation Quality and Testing Robotics (AQTR), 2010, vol. 1, pp. 1-5.

[3] E. Reyes-Valeria, R. Enriquez-Caldera, S. CamachoLara, and J. Guichard, "LQR control for a quadrotor using unit quaternions: Modeling and simulation," in 2013 International Conference on Electronics, Communications and Computing (CONIELECOMP), 2013, pp.
[4] Y. Bouzid, H. Siguerdidjane and Y. Bestaoui, "Real time Autopilot based on Immersion \& Invariance for Autonomous Aerial Vehicle", 20th IFAC Symposium on Automatic Control in Aerospace (ACA), IFACPapersOnLine, vol. 49, no. 17, pp. 176-181, Sherbrooke, Quebec, Canada 21- 25August, 2016.

[5] Y. Bouzid, H. Siguerdidjane and Y. Bestaoui, "Improved 3D trajectory tracking by Nonlinear Internal Model-Feedback linearization control strategy for autonomous systems, " 6th IFAC Symposium on System Structure and Control (SSSC), IFAC-PapersOnLine, vol. 49, no. 9, pp. 13-18, Istanbul, Turkey, 22-24 June 2016.

[6] Y. Bouzid, H. Siguerdidjane, Y. Bestaoui, and M. Zareb, "Energy Based 3D Autopilot for VTOL UAV Under Guidance \&amp; Navigation Constraints," J Intell Robot Syst, pp. 1-22, Nov. 2016.

[7] S. Bouabdallah, P. Murrieri and R. Siegwart, "Design and control of an indoor micro quadrotor," In: proceedings of the IEEE international conference on robotics and automation, 2004. pp.4393-4398.

[8] X. Zhang, X. Li, K. Wang, et al., "A survey of modelling and identification of quadrotor robot," Abstract and Applied Analysis, 2014:1-14.

[9] H. Sira-Ramirez, H.B. Siguerdidjane, "A redundant dynamical sliding mode control scheme for an asymptotic space vehcle stabilization," Int. J. Control, 1996. Vol. 65, No. 6, pp. 901-912.

[10] K. J. Åström, C. C. Hang, and P. Persson, "Towards intelligent PID control," Annual Review in Automatic Programming, vol. 15, pp. 53-58, Jan. 1989.

[11] L. x Ma, D. n Shi, M. x Chen, and X. q Wang, "Application of intelligent PID control for robot," in 2008 IEEE Conference on Cybernetics and Intelligent Systems, 2008, pp. 455-458.

[12] L.Michel, C. Join, M. Fliess, P. Sicard, A. Cheriti, "Model-free control of dc/dc converters," In: 2010 IEEE 12th Workshop on Control and Modeling for Power Electronics, 2010. (COMPEL), pp. 1-8.

[13] J. De Miras, , C. Join, M. Fliess, S. Riachy, S. Bonnet, "Active magnetic bearing: A new step for model-free control," In: 52nd IEEE Conference on Decision and Control, 2013. 